\title{
Contradictions in the Theory of Gas and Hydrodynamics as a Consequence of Incorrectness Fundamental Equations
}

\author{
Vladimir G. Kirtskhalia
}

\begin{abstract}
The author drew attention to the contradictions existing in the theory of gas and hydrodynamics as well as in magnetohydrodynamics as early asin 1994, when he publishedthe article "On the stability problem of the tangential discontinuity" in the journal "Planetary and Space Sciences" [1]. Particularly intensive theoretical research in this direction has been carried out over the past 10 years at I. Vekua Sokhumi Institute of Physics and Technology and at Sokhumi State University. Many works have been published in reputable journals, in which the contradictions and absurdity of some of the concepts ingrainedin modern gas and hydrodynamics have been shown, and ways to overcome them have been indicated. Despite of this, these works did not receive proper feedback from the scientific communityand it can be explained by the conservatism of thinking of specialists in this field of physics. Based on this, we believe that it will be useful to provide interested readers with a review article based on the most important works published by us andonce again todraw their attention to the relevance of the problem.
\end{abstract}

Keywords — fundamental equations, killer wave, speed of sound, wind wave.

\section{INTRODUCTION}

Let us consider the most obvious contradictions that exist in modern gas and hydrodynamics. The most important of them is the determination of the speed of sound in an ideal gas (in air) [2].

$$
C_{s}=\sqrt{\gamma \frac{k T}{m}}
$$

where $\gamma=c_{p} / c_{v}=1.4$ is the ratio of heat capacities at constant pressure and volume, $\mathrm{k}=1.38 \times 10^{-23} \mathrm{~J} /{ }^{\circ} \mathrm{K}$ is the Boltzmann constant, $m=4.81 \times 10^{-26} \mathrm{~kg}$ is the mass of one air molecule, and $T-$ absolute temperature. Formula (1), from which it follows that the speed of sound depends only on temperature and does not depend on altitude, or is the same on the density of the atmosphere is used to calculate the speed of sound in the Earth's atmosphere on any altitude [3]. In other words, the speed of sound at the North Pole at $-40{ }^{\circ} \mathrm{C}$ is the same as in the stratosphere at an altitude of $65 \mathrm{~km}$. The absurdity of this conclusion is obvious because it contradicts the fundamental concepts of physics:

First, the speed of sound is a thermodynamic parameter of any medium and in the Earth's atmosphere, which is a substantially inhomogeneous medium as a result of the effect of the gravitational field on it, it must clearly depend on the spatial coordinate (height).

Secondly, sound represents the propagation of a disturbance o the density of the medium and it is impossible that its speed does not depend on the density itself.

Let us now consider an example of internal gravitational waves, to them $\S 13$ of the monograph [2] is dedicated. The authors call internal waves the waves in an incompressible fluid located in the Earth's gravitational field. Due to incompressibility, they neglect the change in density associated with a change in pressure, and assume that the change in density can only be isobaric, due to a change in entropy, i.e.:

$$
\rho^{\prime}=\left(\frac{\partial \rho_{0}}{\partial s_{0}}\right)_{p} s^{\prime}
$$

Here, the subscript " 0 " denotes the stationary values of the density and entropy, and the prime denotes their perturbed values. As a result, they get a wave, the square of the frequency of which is: 


$$
\omega^{2}=\omega_{0}^{2} \sin ^{2} \theta
$$

where $\omega_{0}$ is some constant frequency and $\theta$ is the angle between the wave vector $\vec{k}$ and the axis $Z$. Thus, the frequency of the internal wave depends only on the direction of the wave vector, the magnitude of which can be any. It is clear that such a wave does not exist in nature and, as will be shown below, the reason for this paradox is the application of the incompressibility condition to the fluid.

I would like to draw attention of the reader to the following controversies related to capillary wavesIn monograph [2], the dispersion equation for a capillary-gravitational wave on the surface of an incompressible fluid is given in the form:

$$
U_{p}^{2}=\left(\frac{\omega}{k}\right)^{2}=\left(\frac{g}{k}+\frac{\alpha k}{\rho}\right) \operatorname{th}(k h)
$$

where $\alpha=0.073 \mathrm{~N} / \mathrm{m}$ is the coefficient of surface tension of water, $\rho=10^{3} \mathrm{~kg} / \mathrm{m}^{3}$, is its density, $g=9,8 \mathrm{~m} / \mathrm{s}^{2}$, is the acceleration of gravity,is the depth of the reservoir. As we can see, it contains gravitational acceleration, although the only stabilizing factor in the boundary condition is the surface tension force. From (4) it follows that,at:

$$
\frac{g}{k}>\frac{\alpha k}{\rho} \Rightarrow k<\sqrt{\frac{\rho g}{\alpha}} \Rightarrow \lambda>2 \pi \sqrt{\frac{\alpha}{\rho g}}=1,72 \mathrm{sm}
$$

The influence of the surface tension force is negligible and the wave becomes purely gravitational. This conclusion is contradicted by the classical experiment in which a steel needle does not sink in a glass filled to the brim with water. It happensbecause although the diameter of the glassis much larger than the length indicated above, there is a surface tension force that balances the pressure generated by the needle. In addition,the presence in (4) of the depth of the reservoiris incomprehensible, because the capillary wave is a purely surface phenomenon and its phase velocity should not depend on the depth.

Another contradiction that we drew attention to is that the equation of motion for a liquid or gas (Euler's equation) contradicts the basic principle of physics, Galileo's principle of relativity, according to which in any inertial frame of reference acceleration must have the same value. Indeed, let's consider the Euler equation in its generally accepted (classical) form:

$$
\rho \frac{d \vec{V}}{d t}=\rho\left[\frac{\partial \vec{V}}{\partial t}+(\vec{V} \nabla) \vec{V}\right]=-\nabla P+\rho \vec{g}
$$

If $\vec{V}_{0}$ is a stationary velocity of motion of a liquid or gas, and $\vec{V}^{\prime}-$ its a small perturbation, then after linearization of equation (6), its left side, which determines the acceleration of a liquid particle, will be equal to $\partial \vec{V}^{\prime} / \partial t+\left(\vec{V}_{0} \nabla\right) \vec{V}^{\prime}$, and thus, the acceleration of a liquid particle depends on the stationary velocity of the medium. This contradiction is so obvious that it is impossible not toadmit it. We see that the presence of a nonlinear term in equation (6) leads to this contradiction.

The purpose of this article is to show that the correct determination of the speed of sound and fundamental equations of gas and hydrodynamics lead to overcoming the above contradictions and clearly and simply explains the dynamic processes occurring in the atmosphere and ocean.

\section{Correct Determination of The SPEedof Sound AND Fundamental Equations of GaS AND HYDRODYNAMICS IN INHOMOGENEOUS MEDIA}

The speed of sound in monograph [2] is determined from the system of two fundamental equations of gas and hydrodynamics:

1) Euler's equation:

$$
\rho \frac{d \vec{V}}{d t}=\rho\left[\frac{\partial \vec{V}}{\partial t}+(\vec{V} \nabla) \vec{V}\right]=-\nabla P+\rho \vec{g}
$$

2) Mass continuity equation: 


$$
\frac{d \rho}{d t}=\frac{\partial \rho}{\partial t}+(\vec{V} \nabla) \rho=-\rho \operatorname{div} \vec{V}
$$

Representing the density and pressure as the sum of their stationary and perturbed values $\rho=\rho_{0}+\rho^{\prime}$, $P=P_{0}+P^{\prime}$ and assuming $\vec{V}$ as a small value, after linearizingequations (7) and (8), they obtain

$$
\left\{\begin{array}{c}
\rho_{0} \frac{\partial \vec{V}}{\partial t}=-\nabla P^{\prime} \\
\frac{\partial \rho^{\prime}}{\partial t}+\rho_{0} \operatorname{div} \bar{V}=0
\end{array} .\right.
$$

From (9) we see that the force of gravity is neglected, assuming that it does not affect the sound, and it is also assumed that the density does not depend on the spatial coordinate. Thus, according to the authors, the atmosphere is a homogeneous ideal gas where the density depends only on pressure and therefore

$$
P^{\prime}=\left(\frac{\partial P}{\partial \rho}\right)_{s} \rho^{\prime}
$$

Introducing the velocity potential $\vec{V}=\operatorname{grad} \varphi$, the system of equations (9) with the use of (10) is reduced to a wave equation of the form:

$$
\frac{\partial^{2} \varphi}{\partial t^{2}}-C^{2} \Delta \varphi=0
$$

where $C=\sqrt{(\partial P / \partial \rho)_{s}}$ is the speed of sound. Applying the law of an ideal gas $P=n k T$ and the dependence of pressure on density at an adiabatic process, $P / P_{0}=\left(\rho / \rho_{0}\right)^{\gamma}$ for the speed of sound they get:

$$
C=\sqrt{\gamma \frac{P}{\rho}}=\sqrt{\gamma \frac{k T}{m}}=\sqrt{\gamma \frac{R T}{M}} .
$$

Here $R=8,314 \mathrm{~J} /{ }^{\circ} \mathrm{Kmol}$ is the gas constant, $M=29 \times 10^{-3} \mathrm{~kg} / \mathrm{mol}$ is the mass of one mole of air. The speed of sound is determined in a similar way in monograph [4].

In the introduction, it was noted that formula (12) does not reflect the properties of the atmosphere and contradicts the fundamental principles of physics. This is due to the fact that the effect of the gravitational field on the Earth's atmosphere is neglected and therefore it is assumed that it is homogeneous. In fact, it is a substantially inhomogeneous medium, in where the density depends on both pressure and entropy $\rho=\rho(P, s)$ and therefore, the density perturbation, in contrast to (10), must be represented in the form:

$$
\rho^{\prime}=\left(\frac{\partial \rho}{\partial P}\right)_{s} P^{\prime}+\left(\frac{\partial \rho}{\partial S}\right)_{p} s^{\prime}
$$

The first term on the right-hand side of (13) describes the change of density as a result of pressure fluctuations at constant entropy or an adiabatic change in the mass of a substance in a constant volume, and the second describes a change in density due to fluctuations in entropy (temperature) at constant pressure, or an isobaric change in the volume of a substance of constant mass. Applying the well-known relations of thermodynamics (for detailed calculations, see [5]), expression (13) can be reduced to the form:

$$
\rho^{\prime}=\left(\frac{1}{C_{s}^{2}}+\frac{1}{C_{p}^{2}}\right) P^{\prime}=\frac{1}{C^{2}} P^{\prime}
$$

(14) is the equation of state of the atmosphere where $C_{s}$ is the adiabatic speed of sound or the speed of 
adiabatic sound expressed by formula (1), $C_{p}$ is the isobaric speed of sound or the speed of isobaric sound which is equal to:

$$
C_{p}(z, T)=\frac{1}{\beta_{p}}\left(\frac{c_{p}}{T}\right)^{1 / 2}=\sqrt{\frac{c_{p} k^{2} T^{3}}{m^{2} g^{2} z^{2}}},
$$

and $C$ is the true speed of sound in the Earth's atmosphere:

$$
C=\sqrt{\frac{C_{s}^{2} C_{p}^{2}}{C_{s}^{2}+C_{p}^{2}}}=\sqrt{\frac{\gamma k T}{m_{0}\left(1+\frac{\gamma m g^{2} z^{2}}{c_{p} k T^{2}}\right)}}
$$

We see that the true speed of sound in the Earth's atmosphere really depends on the altitude (air density), and condition $z=0$ is equivalent to the condition $g=0$, i.e. on the sea level the atmosphere is a homogeneous medium and the speed of sound in it is indeed determined by (1). As the altitude grows, the influence of the Earth's gravitational field increases and the speed of sound decreases.

Another important result follows from (15). As we can see, the coefficient of thermal expansion of air $\beta_{p}$ depends not only on temperature, as it is customary now but also on altitude which refutes the idea of the universality of the laws of an ideal gas. For more details, see [6].

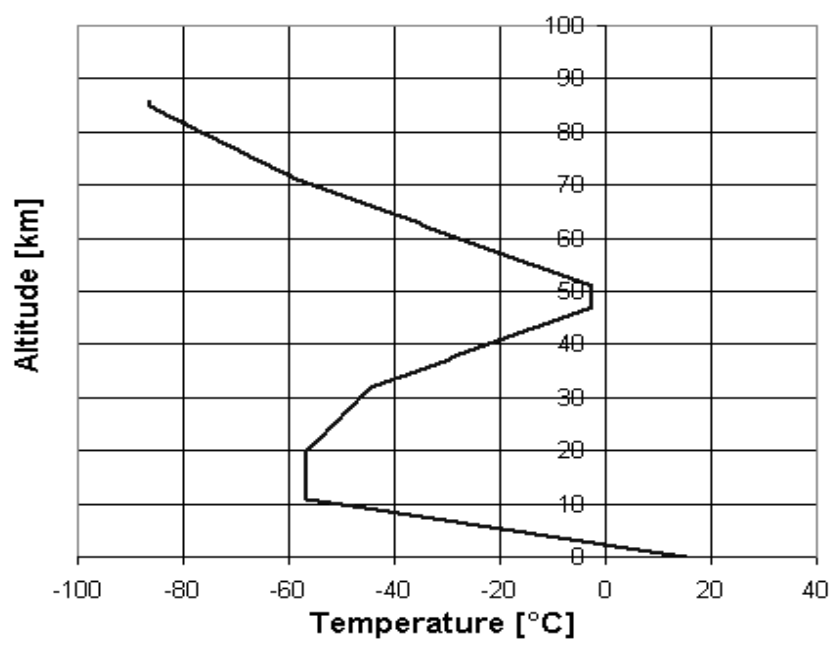

Fig. 1.Temperature as a function of geometrical altitude.

Fig. 1 shows a graph of the temperature distribution according to the height in the Earth's atmosphere, taken from the guide- monograph [7]. We see that from sea level to a height of about $\mathrm{z}=10200 \mathrm{~m}$ the temperature drops according to a strictly linear law $T=\alpha+\beta z$, where $\alpha=288,15^{\circ} \mathrm{K}, \beta=-6,52 \times 10^{-3}{ }^{\circ} \mathrm{K} / \mathrm{m}$. This part of the atmosphere, where there is no heat source and the entropy is constant, is called the troposphere. Thus, our theory is valid only for the troposphere, since it uses the equation of constancy of entropy. At the upper boundary of the troposphere, fall of temperature stops abruptly and remains constant until the height $z=20 \mathrm{~km}$ (tropopause) and then increases (stratosphere). It is known that a sharp change in the dynamics of a physical process is associated with resonance. The resonance of the frequencies of adiabatic and isobaric sounds occurs at $C_{s}=C_{p}$. Equating the right-hand sides of expressions (1) and (15), we obtain:

$$
\frac{\gamma k T}{m}=\frac{c_{p} k^{2} T^{3}}{m^{2} g^{2} z^{2}} \Rightarrow z=\sqrt{\frac{c_{p} k}{\gamma m}} \frac{T}{g}=\frac{\sqrt{c_{p} k \alpha^{2} / \gamma m g^{2}}}{1+\sqrt{c_{p} k \beta^{2} / \gamma m g^{2}}}=10230 \mathrm{~m}
$$

As you can see, the height calculated by the formula (17) practically exactly coincides with the height indicated in Fig. 1, which indicates the high reliability of our theory.

The cessation of the drop in temperature at the upper boundary of the troposphere and its further growth indicate the presence of a certain exothermic processin this region of the atmosphere. In [8], the author assumes that an exothermic reaction of ozone synthesis $\mathrm{O}_{2}+\mathrm{O} \rightarrow \mathrm{O}_{3}+24 \mathrm{kcal} / \mathrm{mol}$ occurs in this area, and 
and we put forward the hypothesis that the resonance $\omega_{s}=\omega_{p}$ is the trigger for this reaction and the reason for such dynamics of the temperature distribution

Although these results, which are of great practical importance, were obtained in 2012 [5], the Internet currently gives the distribution of the speed of sound over the height of the atmosphere calculated by the formula (1) [3]. Table I shows the distribution by height of the values of the adiabatic $C_{s}(T)=C_{\text {int }}(T)$ and true $C(z, T)$ velocities of soundsin the troposphere. There are also given the relative errors between the values of the true and adiabatic sound velocities $(\Delta C / C) 100 \%=\left[\left(C_{s}(T)-C(z, t)\right) / C(z, T)\right] 100 \%$ and the corresponding Mach numbers $\Delta M / M 100 \%=\left\{\left[V / C(z, T)-V / C_{s}(z, T)\right] /[V / C(z, T)]\right\} 100 \%$.

TABLE I: The Altitude Distribution of the VALuES of the Adiabatic AND TRUE Velocities OF SOUND IN THE Troposphere AND THE RELATIVE ERRORS BETWEEN THE TRUE AND AdIABATIC Velocities of SOUND AND THE CORRESPONSING MACH NUMBERS

\begin{tabular}{|c|c|c|c|c|c|}
\hline $\mathrm{Z}(\mathrm{m})$ & $\mathrm{T}^{\circ}(\mathrm{K})$ & $\begin{array}{c}C_{s}=C_{\mathrm{int}}(T) \\
(\mathrm{m} / \mathrm{s})\end{array}$ & $\begin{array}{c}C(z, T)=C(p, T) \\
(\mathrm{m} / \mathrm{s})\end{array}$ & $\frac{\Delta C}{C} 100 \%$ & $\frac{\Delta M}{M} 100 \%$ \\
\hline 0 & 288.15 & 340.29 & 340.28 & 0,0 & 0,0 \\
\hline 1000.00 & 281.65 & 336.43 & 335.45 & 0.29 & 0.29 \\
\hline 2000.00 & 275.15 & 332.53 & 328.47 & 1,22 & 1,22 \\
\hline 3000.00 & 268.65 & 328.58 & 319.34 & 2,89 & 2,81 \\
\hline 4000.00 & 262.15 & 324.58 & 308.12 & 5,31 & 5,07 \\
\hline 5000.00 & 255.65 & 320.53 & 295.04 & 8,62 & 7,95 \\
\hline 6000.00 & 249.15 & 316.43 & 280.42 & 12,79 & 11,38 \\
\hline 7000.00 & 242.65 & 312.27 & 264.66 & 18,02 & 15,25 \\
\hline 8000.00 & 236.15 & 308.06 & 248.18 & 24,1 & 19,44 \\
\hline 9000.00 & 229.65 & 303.79 & 231.38 & 31,35 & 23,84 \\
\hline 10000.00 & 223.15 & 299.46 & 214.65 & 39,49 & 28,32 \\
\hline 11000.00 & 216.65 & 295.07 & 198.26 & 48,83 & 32,81 \\
\hline
\end{tabular}

As we can see, the relative error in determining the Mach number at an altitude of $11 \mathrm{~km}$ is 110 times greater than at an altitude of $1 \mathrm{~km}$. For example, for an airplane flying at a speed of $1000 \mathrm{~m} / \mathrm{s}$ at an altitude of $11 \mathrm{~km}$, the Mach number calculated by formula (4) is equal $M_{1}=1000 / 295,07=3,4$ and by formula (14) $-M_{2}=1000 / 198,26=5,0$. It is obvious that disregard such a large error in calculating the aerodynamic parameters of aircraft is unacceptable (see [9]).

The equation (8) was also obtained for a homogeneous medium, since it determines the change in the density of a substance in a constant volume as a result of the difference between the flows of mass entering and leaving it. Obviously, a change in density is also possible as a result of a change in the volume of a constant mass of a substance, i.e.:

$$
\frac{d \rho}{d t}=\frac{d}{d t}\left(\frac{m}{v}\right)=\frac{v \frac{d m}{d t}-m \frac{d v}{d t}}{v^{2}}=\left(\frac{d \rho}{d t}\right)_{v}-\left(\rho \frac{d}{d t} \ln v\right)_{m}
$$

The first term on the right-hand side of equation (18) is determined by equation (8) and the second term is easily reduced to the form $(\rho d \ln v / d t)_{m}=\vec{V} \nabla P / C_{p}^{2}$. Thus, the equation of continuity of the mass of matter in the gravitational field of the Earth should have the form:

$$
\frac{d \rho}{d t}=-\rho \nabla \vec{V}-\frac{\vec{V} \nabla P}{C_{p}^{2}}
$$

This result radically changes the existing concepts of compressibility and incompressibility of a medium [11]. It is believed that these concepts have a mechanical meaning and do not depend on its thermodynamic properties. In fact, they have a thermodynamic meaning and characterize the homogeneity or inhomogeneity of the medium. A homogeneous medium, where only adiabatic sound propagates, is always compressible. Incompressibility is a consequence of its inhomogeneity, resulting in wich emerage isobaric sound and it is manifested more stronger, the more inhomogeneous is the medium, as it is the case in the Earth's atmosphere with increasing altitude.

This is confirmed by the absurdity of the dispersion equation for internal waves (3), which was obtained under the assumption that the liquid is incompressible $(\nabla \vec{V}=0)$ and neglecting the first term on the righthand side of the mass continuity equation (19). Let's show this with a simple calculation. Substituting in 
(15) the values of the coefficient of thermal expansion and specific heat capacity at constant pressure for water, $\beta_{p}=1,5 \times 10^{-4}{ }^{\circ} \mathrm{K}^{-1}, c_{p}=4,19 \times 10^{3} \mathrm{~J} / \mathrm{kg}^{\circ} \mathrm{K}$, at temperature $T=288^{\circ} \mathrm{K}$ we obtain $C_{p}=25210 \mathrm{~m} / \mathrm{s}$. On the other hand, the speed of sound in water, measured experimentally with great accuracy, and then, from formula (16), we have $C_{s}=C C_{p} / \sqrt{C_{p}^{2}-C^{2}}=1482,6 \mathrm{~m} / \mathrm{s}$. As we can see, the speed of sound in water is practically equal to the adiabatic speed of sound, i.e. $C=C_{s}$. This result irrefutably proves that the mechanism of sound propagation in water is adiabatic $\left(C_{p}=\infty\right)$ and only the first term remains on the right-hand side of (19). Thus, the application of the incompressibility condition to water $\nabla \vec{V}=0$ is not permissible. Moreover, paradoxically, water is a more compressible medium than air in the upper layers of the troposphere, since at an altitude of $10.2 \mathrm{~km}, C_{s} \approx C_{p} \approx 300 \mathrm{~m} / \mathrm{s}$. Thus, the terms "compressibility" and "incompressibility" in thermodynamics characterize not the state of aggregation of a substance, but the physical process of sound propagation in it. "NASA" agrees with this conclusion, since it posted the work [11] in "The Smithsonian / NASA Astrophysics Date System".

We noted above that the classical Euler equations contradict Galileo's principle of relativity. This contradiction is caused by the assumption $d \rho / d t=0 \Rightarrow \rho=$ const, i.e., the assumption of incompressibility of the fluid $\nabla \vec{V}=0$ and neglect of the second term in Eq. (19). In fact, if the first term is zero (incompressible medium), remains the second term, and if the second term is zero (compressible medium), remains the first term. Thus, $d \rho / d t \neq 0$, and in the Euler equation the density must be introduced under the sign of the derivative:

$$
\frac{d(\rho \vec{V})}{d t}=\vec{V} \frac{d \rho}{d t}+\rho \frac{d \vec{V}}{d t}=-\nabla P+\rho \vec{g}
$$

Substituting the value $d \rho / d t$ from equation (19) into equation (20), we get:

$$
\begin{gathered}
-\vec{V} \rho \nabla \vec{V}-\frac{V^{2} \nabla P}{C_{p}^{2}}+\rho \frac{d \vec{V}}{d t}=-\nabla P+\rho \vec{g} \Rightarrow \rho\left[\frac{d \vec{V}}{d t}-(V \nabla) \hat{V}\right]-\frac{V^{2} \nabla P}{C_{p}^{2}}=-\nabla P+\rho \vec{g} \Rightarrow \\
\rho \frac{\partial \dot{V}}{\partial t}=-\left(1+\frac{V^{2}}{C_{p}^{2}}\right) \nabla P+\rho \vec{g}
\end{gathered}
$$

Neglecting the term $V^{2} / C_{p}^{2}$ in comparison with unity, equation (21) takes the form:

$$
\rho \frac{\partial \vec{V}}{\partial t}=-\nabla P+\rho \vec{g}
$$

As we can see, the nonlinear term $(\vec{V} \nabla) \vec{V}$ dropped out of the Euler equation and, therefore, there is no contradiction.

Equation (22) together with equation (19) give the generalized equation of gravitational waves in liquids and gases in the gravitational field of the Earth in the form [12]:

$$
\frac{d^{2} P_{a}(z)}{d z^{2}}+\frac{g}{C^{2}} \frac{d P_{a}(z)}{d z}-\left(k^{2}+\Omega-\frac{\omega^{2}}{C^{2}}\right) P_{a}(z)=0
$$

where $P_{a}(z)$ is the amplitude of the disturbed pressure $P(x, z, t)=P_{a}(z) \exp i(k x-\omega t), C$ is the true speed of sound and $\Omega$ is a certain quantity characterizing the inhomogeneity of the medium. Calculations show [12] that in the troposphere $\bar{\Omega}_{1}=-8.2 \times 10^{-9} \mathrm{~m}^{-2}$ and since in the sound wave $P_{a}=$ const and $k^{2}>>\bar{\Omega}$, from equation (23) we obtain:

$$
k^{2}-\frac{\omega^{2}}{C^{2}(z)}=0
$$

Equation (24) is the dispersion equation of a sound wave propagating in the horizontal direction, and 
from it appearsthat the speed of sound in the troposphere clearly depends on $z$ according to formula (16).

Now about capillary waves. A capillary wave is generated and propagates along the plane surface of the water-air interface, and obtaining its dispersion equation is a typical problem of tangential discontinuity. Therefore, two equations are written for air $\mathrm{z}>0$ and for water $\mathrm{z}<0$.

$$
\begin{gathered}
\frac{d^{2} P_{a 1}(z)}{d z^{2}}+\frac{g}{C_{1}(0)} \frac{d P_{a 1}(z)}{d z}-\left[\frac{\omega}{C_{1}^{2}(0)}\left(\omega-k V_{0}\right)-k^{2}\right] P_{a 1}(z)=0 \\
\frac{d^{2} P_{a 2}(z)}{d z^{2}}+\frac{g}{C_{2}} \frac{d P_{a 2}(z)}{d z}-\left[\frac{\omega^{2}}{C_{2}^{2}}-k^{2}\right] P_{a 2}(z)=0
\end{gathered}
$$

It is assumed here that air moves relative to water at a speed $\vec{V}_{0}$ and it is taken into account that the atmosphere at sea level (where the capillary wave propagates) and water are homogeneous media, and therefore $\Omega_{1}=\Omega_{2}=0$. The solutions of these two equations are stitched together on the surface of the tangential discontinuity $z=0$ by the boundary condition:

$$
\left.\left(P_{2}-P_{1}\right)\right|_{z=0}=-\alpha \frac{\partial^{2} \xi}{\partial x^{2}}
$$

where $\xi=\xi_{0} \exp i(k x-\omega t)$ is the displacement of the surface along the axis $Z$, and then we obtain the dispersion equation of the capillary wave, whose solution is [13]:

$$
U_{p}=\frac{\omega}{k}=\frac{-\rho_{01} V_{0} \pm \sqrt{\rho_{01}^{2} V_{0}^{2}+4 \alpha k \rho_{02}}}{2 \rho_{02}} \text {. }
$$

We see that in formula (28) there are no contradictions noted by us above. Namely:

1) The equation does not contain gravitational acceleration and, thus, there are no capillarygravitational waves and, therefore, there are no the conditions limiting the length of the capillary wave.

2) Since $\rho_{01}^{2} V_{0}^{2}+4 \alpha k \rho_{02}>0$ for any $k$, contrary to the Kelvin stability condition [2], the capillary wave is stable in any wind, unless the wind force exceeds the intermolecular interaction force, at which the problem statement becomes meaningless.

3) The phase velocity of the capillary wave does not depend on the depth of the reservoir.

Our theory gives the most tangible results while considering surface gravitational waves, which is like the problem of capillary wavesa problem of tangential discontinuity with the only difference that the equation for air is written in the form (23), where $C_{l}$ and $\Omega_{1}$ are averaged over the coordinate $z$ within the boundaries of the troposphere and instead of boundary condition (27), it is written [12]:

$$
\left.P_{2}\right|_{z=0}=\left.P_{1}\right|_{z=0}+\rho_{02} g \varsigma(x, t) .
$$

This approach makes it possible to clearly classify waves of wind and tsunamis which is absent in the existing literature. Namely, wind waves are waves whose lengths satisfy the condition

$$
k>9,05 \times 10^{-5} / \sqrt{1-x^{2}} m^{-1} \Rightarrow \lambda<6.94 \times 10^{4} \sqrt{1-x^{2}} m
$$

where $x=U_{p} / \bar{C}_{1}$. Otherwise, we have a tsunami wave. For wind waves, the dispersion equation breaks down into two independent equations, whose solutions are:

$$
\left|U_{p}\right|=\sqrt{1-\frac{1,17 \times 10^{-8}}{k^{2}}} \bar{C}_{1}=\sqrt{1-\frac{1,17 \times 10^{-8} \lambda^{2}}{4 \pi^{2}}} \bar{C}_{1}
$$




$$
\left|U_{p}\right|=\sqrt{\frac{g}{k} \tanh (k H)}=\sqrt{\frac{g \lambda}{2 \pi} \tanh \left(\frac{2 \pi H}{\lambda}\right)} .
$$

Expression (31) determines the phase velocities of longitudinal waves in the atmosphere generated by waves on the water surface. We see that they do not depend on the depth of the ocean and at $k>0.34 \mathrm{~m}^{-1} \Rightarrow \lambda<18.5 \mathrm{~m}$ are constants and equals $U_{p}=\bar{C}_{1} \cong 281,72 \mathrm{~m} / \mathrm{s}$ and then, with decreasing $k$ they fall and at $k=1.08 \times 10^{-4} \mathrm{~m}^{-1} \Rightarrow \lambda=5.81 \times 10^{4} \mathrm{~m}$ we have $U_{p}=0$. i.e. the wave stops. This means that at such a wavelength in the atmosphere above the water, two regions with a length of about $30 \mathrm{~km}$, with high and low pressures are formed. In the region of low atmospheric pressure, the amplitude of the surface wave will increase, and the pressure difference between the two regions will lead to the occurrence of wind. With a further decrease $k$ to its minimum value $k_{\min } \cong 9 \times 10^{-5} \mathrm{~m}^{-1} \Rightarrow \lambda_{\max } \cong 7 \times 10^{4} \mathrm{~m}$ which is determined from (30), the roots of equation (31) and the corresponding frequencies of standing waves in the atmosphere become imaginary, which leads to a sharp increase in the pressure difference and, consequently the amplitude of the surface wave and of wind force. This result clearly explains the reason of the drop in atmospheric pressure over the sea and ocean before the storm starts, as well as the reason of its strengthening. Expression (32) coincides with the well-known expression from which the phase velocities of surface gravitational waves are calculated.

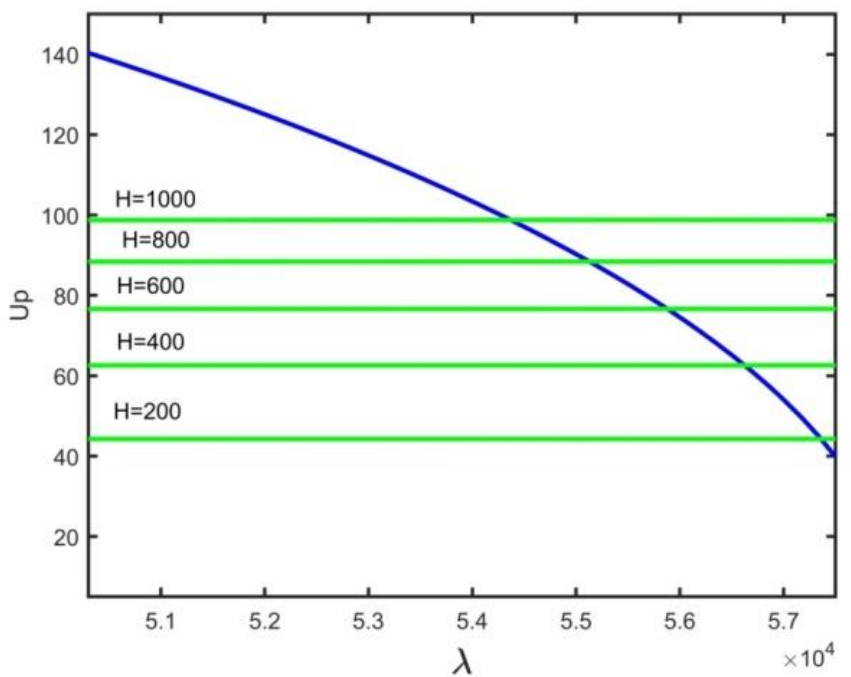

Fig. 2. Plots of dependences of the phase velocities of longitudinal waves in air (80) (blue curve) and on the water surface (82) (green curves) for different depths.

Fig. 2 shows the graphs of dependense the phase velocities on wavelength in the atmosphere (blue curve) and in water for different depths (green lines). We see that they intersect at different wavelengths at different depths. For these valuesand, the resonance of the frequencies of the atmospheric longitudinal wave and the surface gravitational wave occurs. If, at the same time, oscillations in these waves occur in antiphase, that is, if there is a rarefaction area in the atmosphere and a thickening area under it in the ocean, a sharp increase in the amplitude of the surface wave will occur and a "killer wave" will appear. Thus, our theory provides a simple and visual explanation of the phenomenon which is often called the "great secret of nature". In addition, our theory shows that according to observations, the tsunami wave does not affect the atmosphere.

\section{CONCLUSION}

A brief review of our research and their results show that the new approach to the problems of gas and hydrodynamics proposed by us clearly and logically explains the qualitative and quantitative aspects of many known physical phenomena occurring in nature. Despite this, our works were fundamentally rejected by authoritative specialized journals that are obliged to support and popularize, orpresented to readers for discussion, original articles in which new results were obtain differed from well-known, well-established ideas and concepts. The deviations were due to negative reviews given by reviewers who are highly likely to be leading experts in this field of physics. Here we will give a few quotes from the reviewers' reviews that have survived with us and let the reader judge their objectivity for himself.

To the classic experience which we cited, concerning a steel needle that does not sink in a glass of water, one of the reviewers objected, "here the diameter of the glass does not play any role, the needle does not sink because its diameteris less than the specified length". 
It is especially surprising that the reviewers do not even try to grasp the essence of the problem and understand why our results so convincingly and simply eliminate the obvious contradictions existing in modern gas and hydrodynamics and explain many natural phenomena. For example, one of the reviewers of work [12] writes - "The author should explain in detail why this study is necessary. Is it just fun for applied mathematicians or useful to the geoscience community? "Or - " The authors tried to present a new dispersion relationship for water waves by taking into account the effects of 'air motions'. However, the dispersion relationship for air and that for water are, at the end of the day, split into two independent relations. This fact means that there is no interaction between air and water". Another reviewer writes"Reviewer has encountered the novel speed of isobaric sound, which results from the equation of continuity derived. Even for internal waves in stratified in homogeneous fluids, however, the equation of continuity is given universally by the one without the last term proportional to the pressure gradient. This result contradicts the adiabatic sound speed widely accepted", or- "The homogeneous medium is always compressible. The incompressibility is a consequence of its non-homogeneity ..." ". This is beyond the Reviewer's understanding. From the viewpoint of the standard fluid mechanics, this paper is unsuitable for publication in the Journal of Fluid Mechanics.

We are citing these quotes here not to question the competence of their authors, but only to show how firmly dogmas and concepts that have a two-century history sit in the minds of modern scientists. It is clear that these dogmas and concepts are associated with the names of such great scientists as: Kelvin, Helmholtz, Euler and others, butweshould note that the works of these scientists also contain errors, discussed in detail in [14].

It follows from the above that the problems we have posed and their solutions require serious discussion in the scientific community. We believe that the publication of this article in the European Journal of Applied Sciences will facilitate this process.

\section{REFERENCES}

[1] V. Kirtskhalia. On the stability problem of the tangential discontinuity, Planet. Space Sci. 1994; 42(6):513-518.

[2] Landau L.D. andLifchitz E.N. Theoretical Physics. Hydrodynamics, 1988, vol. 6, Moscow, "Nauka".

[3] http://www.sengpielaudio.com/calculator-speedsound.htm.

[4] Earl E. Gossard and William H. Hooke, Waves in the Atmosphere, 1975.

[5] V. G. Kirtskhalia. Speed of Sound in Atmosphere of the Earth. Open Journal of Acoustics, 2012;2(2):80-85.

[6] V.G. Kirtskhalia. On Universality of Laws of Ideal Gases. Journal of Modern Physics 2015;7:948-954. http://dx.doi.org/10.4236/jmp.2015.67099.

[7] U.S. Standard Atmosphere. 1976. (National Aeronautics and Space Administration).

[8] O.G. Sorokhtin. The process of absorption of ultra-violet radiation of the Sun by terrestrialatmosphere. Bulletin of the Russian Academy of Natural Sciences, 2009/3.

[9] V.G. Kirtskhalia. The dependence of the speed of sound in the Earth's atmosphere on its density and the correction of Mach's number. IOP Conf. Series: Materials Science and Engineering, 1024 (2021) 012037 doi:10.1088/1757-899X/1024/1/012037.

[10] V.G. Kirtskhalia. Sound wave as a particular case of the gravitational wave. Open Journal of Acoustics, 2012;2(3):115-120. doi.org/10.4236/oja.2012.23013.

[11] V.G. Kirtskhalia. The New Determination of the Criteria of Compressibility and Incompressibility of Medium. Journal of Modern Physics, 2013;4(8):1075-1079. doi.org/10.4236/jmp.2013.48144.

[12] V. G. Kirtskhalia, K. R. Ninidze. Mutual Influence of the Atmosphere and the Ocean under Wave Processes. Journal of Modern Physics, 2021;12:1346-1365. https://doi.org/10.4236/jmp.2021.129081.

[13] V.G. Kirtskhalia. The Linearity of the Euler Equation as a Result of the Compressibility of a Fluid. Journal of Modern Physics, 2019;10(4):452-458. doi.org/10.4236/ jmp. 2019.104030.

[14] V.G. Kirtskhalia. Correct Definition of Sound Speed and its Consequences in the Tasks of Hydrodynamics. Journal of Fluids, vol. 2016, ID 4519201, 9 pages. http://dx.doi.org/10.1155/2016/4519201. 